\title{
Strates
}

STRATES Matériaux pour la recherche en sciences sociales

9 | 1997

Crises et mutations des territoires

Statistiques inédites

\section{Les salariés des exploitations agricoles dans les élections aux Chambres d'agriculture}

Jocelyne Hacquemand et Françoise Plet

(2) OpenEdition

Journals

Édition électronique

URL : http://journals.openedition.org/strates/641

DOI : $10.4000 /$ strates.641

ISSN : $1777-5442$

Éditeur

Laboratoire Ladyss

Édition imprimée

Date de publication : 30 septembre 1997

ISSN : 0768-8067

Référence électronique

Jocelyne Hacquemand et Françoise Plet, «Les salariés des exploitations agricoles dans les élections aux Chambres d'agriculture », Strates [En ligne], 9 | 1997, mis en ligne le 19 octobre 2005, consulté le 08 septembre 2020. URL : http://journals.openedition.org/strates/641 ; DOI : https://doi.org/10.4000/ strates.641

Ce document a été généré automatiquement le 8 septembre 2020

Tous droits réservés 


\title{
Statistiques inédites
}

\section{Les salariés des exploitations agricoles dans les élections aux Chambres d'agriculture}

\author{
Jocelyne Hacquemand et Françoise Plet
}

1 Les élections du collège des salariés aux chambres d'agriculture sont particulièrement mal connues, ignorées des médias et peu étudiées par les chercheurs. L'ampleur nouvelle que prend le salariat agricole et péri-agricole dans les activités de cette sphère de production mérite la diffusion de données sur les électeurs et le comportement électoral de cette catégorie professionnelle, en dépit du caractère très minoritaire de sa représentation au sein des Chambres.

2 Le régime électoral dont sont issues les Chambres d'agriculture s'est constitué progressivement. En 1961, quatre collèges sont représentés : les exploitants et leur famille, les salariés agricoles, les propriétaires non exploitants, les organisations agricoles. En 1963, les administrateurs élus des Comités régionaux de la propriété forestière deviennent membres des Chambres d'agriculture. En 1969, un collège d'anciens exploitants est créé, tandis que les salariés d'organismes sont inclus dans le collège des salariés agricoles, ce qui traduit à la fois le développement de l'emploi périagricole, et la volonté de renforcer le collège des actifs agricoles par celui des retraités. En 1973, le collège des salariés est divisé en collège des salariés des exploitations et collège des salariés des organismes. Le nombre des sièges n'a pas augmenté en dépit de la progression du nombre de salariés agricoles. Ces deux collèges représentent en effet près de 420000 salariés inscrits sur les listes électorales (cf. tableau 1).

3 Le code rural établit actuellement dix collèges :

$4 \quad 1:$ chefs d'exploitation et assimilés (21 sièges)

52 : propriétaires et usufruitiers (2 sièges)

$6 \quad 3 \mathrm{~A}$ : salariés d'exploitation (4 sièges)

7 3B : salariés d'organismes (coopératives, Crédit Agricole, MSA, OPA, Institut) (4 sièges) 

pour les organisations syndicales de salariés en agriculture. Elles déterminent la place de chaque organisation dans les instances de discussion et de décision, mettant en présence pouvoirs publics, organisations professionnelles agricoles et organisations syndicales à tous les niveaux territoriaux. Ainsi le préfet désigne-t-il le représentant de la première organisation syndicale de salariés pour siéger à la Commission Départementale d'Orientation Agricole (CDOA) instituée par la loi de modernisation de l'agriculture à l'automne 1994.

Deux collèges donc concernent les salariés de l'agriculture. Le collège $3 \mathrm{~A}$ est celui des salariés d'exploitations agricoles, forestières et sylvicoles et des salariés de l'office National des Forêts (ONF) sous statut privé. C'est celui pour lequel nous donnons des résultats électoraux détaillés. Le collège $3 \mathrm{~B}$ est le plus important en terme de nombre de salariés concernés. Il est constitué des salariés d'organismes, coopératives, organismes de développement, caisses de Crédit Agricole, Mutualité Sociale Agricole, salariés de l'institution des Courses hippiques. Sont électeurs les salariés ressortissants 
des États membres de l'Union Européenne, affiliés aux Assurances sociales agricoles et remplissant les conditions d'activité professionnelle exigées pour l'ouverture de droits aux prestations de l'Assurance maladie.

L'inscription sur les listes électorales est une inscription volontaire de chaque électeur. La mairie doit publier les listes, établies suivant la précédente élection et sur demande individuelle des salariés. Certaines organisations syndicales de salariés ont demandé l'inscription automatique par l'employeur, proposition jusqu'ici rejetée par le ministère de l'Agriculture.

Le Code rural prévoit le vote des salariés sur leur temps de travail. Souvent, les exploitants ne donnent pas à leurs salariés l'autorisation d'absence au moment du vote. L'éparpillement des salariés des exploitations dans l'espace, tout comme leur faible syndicalisation, ne permettent guère d'imposer aux employeurs le respect du droit de vote. Malgré un taux moyen de participation de $28 \%$ (collèges 3A et 3B) lors des dernières élections, ce sont près de 117000 salariés qui se sont exprimés. La participation un peu plus active des salariés du collège 3B, 30,2 \% en 1995 contre $24 \%$ pour le collège $3 \mathrm{~A}$, est sans doute liée à une présence plus importante des organisations syndicales grâce à une concentration plus forte des salariés sur les lieux de travail. Mais la représentation des salariés dans les chambres d'agriculture reste peu connue de la population concernée, les seuls collèges exploitants étant médiatisés. Il existe, de plus, une difficulté réelle des salariés à percevoir leur apport à l'orientation et au développement des Chambres². (cf. en annexe : Les chambres d'agriculture)

Les résultats confèrent une forte représentativité de la CFDT et de la CGT (près de $60 \%$ de votants). Les suffrages de ces deux organisations progressent par rapport aux élections de 1989 , de $29,76 \%$ à $31,12 \%$ des suffrages exprimés en 1995 pour la CFDT et de $25,82 \%$ à $27,64 \%$ pour la CGT. Troisième organisation, FO atteint $14,79 \%$ des voix et régresse légèrement par rapport à 1989 . La CGC se stabilise à 10,27\%. Le nombre des présentées par les organisations syndicales est en augmentation: 232 listes départementales en 1995 contre 179 en 1989. Les listes communes qui réunissaient 10,56 \% des suffrages en 1989 n'en représentent plus que 4,8 \% en 1995.

23 En 1995, la CFDT reste la première organisation syndicale dans 9 régions : elle perd le Nord-Pas-de-Calais au profit de FO mais maintient sa domination dans le Grand Ouest avec la majorité absolue en Bretagne et Pays-de-Loire. La CGT, première organisation syndicale dans 6 régions, gagne la Provence-Alpes-Côte-d'Azur, mais elle perd le Limousin. FO devient la première organisation dans le Limousin $(52,48 \%)$ et le NordPas-De-Calais (36,13 \%) au détriment de la CFDT. La CGC gagne en 1995, la Corse et l'Ile de France.

Tableau 1 : Collèges salariés : élections 1989 et 1995

\begin{tabular}{|l|l|l|l|l|}
\hline & 1989 & 1989 & 1995 & 1995 \\
\hline \hline & $3 \mathrm{~A}$ & $3 \mathrm{~B}$ & $3 \mathrm{~A}$ & $3 \mathrm{~B}$ \\
\hline \hline Inscrits & 169422 & 249539 & 160277 & 258975 \\
\hline \hline Votants & & & \\
\hline
\end{tabular}


\begin{tabular}{|l|l|l|l|l|}
\hline Taux de participation & $29,89 \%$ & $35,97 \%$ & $23,97 \%$ & $30,22 \%$ \\
\hline
\end{tabular}

Source : ministère de l'Agriculture

Les salariés d'exploitation

En 1960 les salariés assuraient environ $20 \%$ du travail total de la production agricole. Entre 1979 et 1988 cette part était à son minimum historique, aux environs de $15 \%$. Depuis 1990, la part des salariés permanents et saisonniers a augmenté pour atteindre 18 \% du travail total (source SCEES) en 1994.

La MSA comptait en 1993 dans les exploitations agricoles 126238 salariés permanents ${ }^{3}$ et 562331 saisonniers, dont 227263 travaillant entre 20 et 199 jours dans l'année. Au total, 688569 salariés ont travaillé chez 116113 employeurs. En «équivalent temps plein» ${ }^{4}$, le nombre de salariés atteint 206958 . Dans l'ensemble du secteur de la production agricole, qui concerne également les professions hippiques, les travaux forestiers, les entreprises de travaux agricoles et les entreprises paysagistes, le nombre total des salariés atteint 841532 pour 298821 «équivalents temps plein»5.

L'enquête sur les structures menée par le SCEES en 1993 permet d'affiner l'analyse du salariat en agriculture. 62450 entreprises agricoles $(7,8 \%)^{6}$ employaient 136202 salariés permanents. Les exploitations avec salariés permanents détiennent le quart du potentiel agricole français. Les exploitations de grande taille (plus de 10 salariés permanents) occupent $22 \%$ des salariés permanents tout en représentant à peine plus de $2 \%$ des entreprises agricoles employeuses de main-d'œuvre. La croissance du poids relatif du salariat dans l'emploi agricole total tient à la croissance de l'emploi salarié dans les grandes entreprises agricoles : les entreprises de plus de 5 salariés employaient $34 \%$ de la main-d'œuvre salariée en 1979 et $38 \%$ en 1993 (CETA et CUMA compris). $69 \%$ des salariés équivalent temps plein sont employés en cultures spécialisées (maraîchage, horticulture...) et en viticulture. Les salariés permanents sont essentiellement employés par les exploitations viticoles, fruitières, horticoles. Suivent les exploitations de grandes cultures. Les exploitations d'élevage emploient peu de salariés permanents.

Mais la précarité de l'emploi agricole salarié augmente: en 1993, dans la production agricole, on compte 2,3 salariés occasionnels pour 1 salarié permanent. 454000 salariés (52,6 \% des salariés de la production agricole) ont travaillé moins de 40 jours, dont $80 \%$ moins de 20 jours. 210000 salariés $(24 \%)$ ont travaillé entre 40 et 200 jours dans l'année : ils sont considérés comme intermittents. 199000 salariés ont travaillé au moins 200 jours en 1993 : parmi eux plus des trois quarts sont à temps complet (+ de 240 jours). Cette population représente $23 \%$ des salariés de la production agricole.

Entre 1984 et 1993, le salariat de la production évolue selon trois types de modalités : le nombre des salariés augmente tandis que le nombre des "équivalents temps plein » (ETP) stagne ou diminue dans les grandes cultures et l'élevage, ce qui exprime un recours plus important au travail intermittent ou occasionnel en même temps qu'une meilleure déclaration des salariés ; au contraire, tous les types d'emploi se réduisent de manière importante dans les travaux forestiers $(-20 \%)$ et l'artisanat rural $(-39 \%)$; enfin, le nombre des salariés et des ETP augmente dans les entreprises paysagistes (+45\%), dans la conchyliculture, les activités hippiques et les services.

Ces évolutions au niveau de la production révèlent particulièrement bien les mutations en cours dans les activités rurales liées à l'agriculture, avec le développement d'emplois 
de service, et la substitution partielle du salariat au travail familial dans des exploitations agricoles moins nombreuses, en moyenne plus grandes et de moins en moins individuelles: les exploitations de forme sociétaire représentent $33 \%$ des employeurs de main-d'œuvre.

Tableau 2 : Les salariés de l'agriculture (1993)

\begin{tabular}{|c|c|c|c|c|c|c|}
\hline \multirow[t]{2}{*}{$\begin{array}{c}\text { Secteurs } \\
\text { de production }\end{array}$} & \multirow[t]{2}{*}{$\begin{array}{c}\text { Nombre } \\
\text { d'employeurs }\end{array}$} & \multicolumn{3}{|c|}{$\begin{array}{c}\text { Nombre de salariés en fonction } \\
\text { du nombre de jours } \\
\text { de travail par an }\end{array}$} & \multirow[t]{2}{*}{$\begin{array}{c}\text { Nombre } \\
\text { total } \\
\text { de salariés }\end{array}$} & \multirow[t]{2}{*}{$\begin{array}{l}\text { Nombre } \\
\text { d'équivalents } \\
\text { temps plein }\end{array}$} \\
\hline & & $\begin{array}{l}\text { MOINS DE } \\
20 \text { JOURS }\end{array}$ & $\begin{array}{l}\text { DE } 20 \mathrm{~A} \\
199 \text { JOURS }\end{array}$ & $\begin{array}{l}200 \text { JOURS } \\
\text { ET PLUS }\end{array}$ & & \\
\hline $\begin{array}{l}\text { Cultures } \\
\text { spécialisées }\end{array}$ & 21.201 & 82.201 & 107.276 & 44.993 & 234.470 & 79.844 \\
\hline $\begin{array}{l}\text { Elevages } \\
\text { spécialisés }\end{array}$ & 5.920 & 6.001 & 12.640 & 12.275 & 30.916 & 17.119 \\
\hline Viticulture & 32.513 & 159.496 & 48.905 & 33.840 & 242.241 & 54.033 \\
\hline $\begin{array}{l}\text { Cultures et } \\
\text { élevages non } \\
\text { spécialisés }\end{array}$ & 56.479 & 87.370 & 58.442 & 35.130 & 180.942 & 55.962 \\
\hline $\begin{array}{l}\text { Sous-total } \\
\text { Exploitations } \\
\text { agricoles }\end{array}$ & 116.113 & 335.068 & 227.263 & 126.238 & 688.569 & 206.958 \\
\hline $\begin{array}{l}\text { Entrainement, } \\
\text { dressage } \\
\text { de chevaux }\end{array}$ & 4.072 & 2.443 & 9.319 & 5.290 & 17.052 & 8.883 \\
\hline $\begin{array}{l}\text { Travaux } \\
\text { forestiers }\end{array}$ & 8.364 & 4.252 & $17 / 747$ & 24.380 & 46.379 & 32.202 \\
\hline $\begin{array}{l}\text { Entreprises } \\
\text { de travaux } \\
\text { agricoles }\end{array}$ & 6.997 & 6.651 & 13.154 & 10.584 & 30.389 & 15.292 \\
\hline $\begin{array}{l}\text { Entreprises } \\
\text { Paysagistes }\end{array}$ & 7.480 & 5.578 & 19.152 & 24.137 & 48.867 & 31.901 \\
\hline $\begin{array}{l}\text { Conchyliculture } \\
\text { marais salants }\end{array}$ & 1.557 & 4.139 & 3.916 & 2.221 & 10.276 & 3.585 \\
\hline $\begin{array}{l}\text { Total } \\
\text { Production } \\
\text { Agricole }\end{array}$ & 144.583 & 358.131 & 290.551 & 192.850 & 841.532 & 298.821 \\
\hline
\end{tabular}

Source : Observatoire économique et social de la MSA

\section{ANNEXES}

\section{Les Chambres d'agriculture}

Organismes consultatifs, les Chambres d'agriculture disposent de moyens d'action importants.

L'idée d'instituer un système de représentation publique des agriculteurs est née au milieu du XIX ${ }^{\mathrm{e}}$ siècle. C'est finalement la loi du 3 janvier 1924 qui institua dans chaque département une Chambre d'agriculture, institution professionnelle élue au suffrage universel dotée du statut d'établissement public. Les premières élections n'eurent lieu qu'en 1927. A l'issue de la Seconde guerre mondiale, ce n'est qu'en 1949, sous l'impulsion de la FNSEA (Fédération Nationale des Syndicats Agricoles) que renaissaient les Chambres, élues par tous les «intérêts agricoles». Un accord (8 février 1951) 
subordonnait les chambres au syndicat : elles s'engageaient à ne donner d'avis aux pouvoirs publics qu'après consultation des fédérations d'exploitants. Mais très rapidement elles s'exprimeront indépendamment des options de la FNSEA.

Les Chambres furent progressivement dotées de fonctions de participation au développement économique doté de moyens équivalents dans l'ensemble des départements grâce au Fonds National de Péréquation des Chambres d'Agriculture (décret-loi du 24/11/1954). L'un des outils en est la vulgarisation agricole avec des services techniques rendus obligatoires en 1966. Ils emploient en 19962000 conseillers agricoles. La même année, les Établissements départementaux d'élevage (loi du 28/12/1966) sont chargés de l'amélioration technique du secteur pour la plupart constitués comme service des Chambres d'agriculture. Les Chambres participent à de multiples instances consultatives, comme la commission des structures agricoles ou le comité de la formation professionnelle. Elles proposent et soutiennent des projets dans des domaines variés tels le développement de nouvelles filières de production, l'aménagement, la recherche de débouchés, le tourisme rural, ce qui les conduit à jouer un rôle de coordination entre les différents partenaires du développement du monde agricole et rural. Elles sont un centre de services aux agriculteurs, services qui s'étendent du foncier à l'économie, à la gestion, voire à l'expérimentation et à la recherche. Elles peuvent se concerter pour créer des Services d'Utilité Agricole à Compétence Interdépartementale (S.U.A.C.I.), ce qui leur confère un pouvoir important au travers de leurs 4500 élus et de leurs 6200 salariés.

La politique de maîtrise des productions et de réduction des garanties inscrite dans la réforme de la Politique Agricole Commune de 1992 ouvre une nouvelle phase dans l'histoire du développement agricole. Bien que placé au sein d'un organisme consulaire élu, le développement reste un enjeu de pouvoir pour la FNSEA et le CNJA. Depuis sa création, il a été l'objet de tentatives voire de prises de contrôle d'instruments de sa maîtrise, tels les groupes de vulgarisation (GVA, GDA, CETA) par la FNSEA. Le syndicalisme a également contrôlé la mise en œuvre des plans de développement créés en 1972 par la CEE pour accélérer la croissance d'exploitations modernes. Au début des années 1980, avec la mise en place progressive de mesures de réduction de la production subventionnée, la FNSEA revendique une diminution des taxes parafiscales. Une réforme du financement de l'ANDA est en discussion. Cette réforme aura obligatoirement des répercussions sur l'ensemble des instituts techniques rattachés aux filières de production, organismes à vocation agricole générale ${ }^{7}$, et Chambres d'agriculture.

Les ressources des Chambres sont la taxe foncière sur les propriétés non bâties, les participations du Fonds National de Développement Agricole, des Conseils Généraux et Régionaux, de l'État et les redevances des utilisateurs de leurs prestations de services.

\section{NOTES}

1. Qui désignent à l'échelon cantonal les représentants des salariés dans les caisses de la MSA. Les dernières se sont déroulées en décembre 1994.

2. Le taux de participation aux élections des représentants des salariés dans les caisses de Mutualités Sociales Agricoles est également très faible : 28,6 \% en 1994 
3. Cette partie statistique a été réalisée à partir de deux sources : le Service Central des Enquêtes et Études statistiques du ministère de l'Agriculture et de la Pêche (SCEES) et l'Observatoire économique et social mis en place par la MSA (Mutualité Sociale Agricole) en 1992. Leurs données, sensiblement différentes doivent être analysées avec précautions. Les statistiques de la MSA sont réalisées à partir de la déclaration des exploitations. La MSA définit comme «salariés permanents » ceux qui ont travaillé plus de 200 jours par an ; un salarié " équivalent à temps plein » correspond à 2028 heures de travail par an. Les salariés intermittents ont travaillé entre 40 et 200 jours dans l'année, les salariés occasionnels moins de 40 jours. Les statistiques du ministère relèvent d'enquêtes et utilisent la notion d'UTA (unité-travail-année) qui correspond à 2200 heures de travail par an. Toutefois, ces deux sources relèvent les mêmes tendances.

4. Nombre total des heures travaillées divisé par 2028.

5. Les exploitants agricoles sont 900000 à la même date.

6. Le SCEES inclut les ETA [Entreprises de Travaux Agricoles] et les CUMA [Coopératives d'Utilisation du Matériel Agricole].

7. Tels la CFCA (Confédération Française de la Coopération Agricole), la FNSEA, le CNJA.

\section{RÉSUMÉS}

Les élections aux Chambres d'agriculture des représentants du collège des salariés d'exploitations agricoles sont beaucoup plus mal connues que celles du collège des chefs d'exploitation. Or la place du salariat progresse à nouveau dans le travail agricole. Les tableaux présentés fournissent les résultats complets par départements de 1989 à 1995.

\section{Polling for agriculture wage earner's representatives}

Polling for Chambres d'agriculture's cultivators deputies are closely studied. On the opposite, polling for agriculture wage earner's representatives are almost ignored though their place is growing in agricultural employment. These charts give detailed figures at departement level for 1989's and 1995's polls.

\section{INDEX}

Mots-clés : salariés agricoles, chambres d'agriculture

Keywords : agriculture wage earners

\section{AUTEURS}

\section{JOCELYNE HACQUEMAND}

Jocelyne HACQUEMAND est doctorante au sein du laboratoire LADYSS Elle travaille à une thèse consacrée à Béghin-Say et l'Europe. 
FRANÇOISE PLET

Françoise PLET est professeur de géographie à l'Université Paris VIII. Ses travaux de recherche portent sur les interactions entre agricultures et agroindustries et leurs effets territoriaux. Dans le cadre du laboratoire LADYSS, elle est responsable d'un programme de recherche sur la région viticole de Tokaj (Hongrie). 